\title{
Claves epistemológicas para la formación de investigadores educativos
}

\section{Epistemological keys for training educational researchers}

José Ángel Bermúdez García

Pontificia Universidad Católica del Ecuador, Ecuador

Paulina de los Ángeles Morales Hidalgo

Pontificia Universidad Católica del Ecuador, Ecuador

Jean Carlos García Zacarías

Pontificia Universidad Católica del Ecuador, Ecuador

Autor para correspondencia: bjoseangel@yahoo.es, pmorales048@puce.edu.ec, jcgarciazac@yahoo.es

Fecha de recepción: 28 de Agosto 2017 - Fecha de aceptación: 15 de Enero de 2018

Resumen: la formación para praxis científica de los investigadores educativos de nuestra Universidad se desarrolla bajo la mentalidad positivista, promoviéndose una cultura educativa de la férrea lógica instrumental y recetaría. Queda al descubierto la grave crisis epistemológica, un vacío ontológico y antropológico a la hora de comprender la realidad. Esta investigación se acerca existencialmente a las claves epistemológicas que pueden contribuir a pensar los procesos formativos y la praxis científica de los investigadores educativos para inscribirlos en el camino de la nueva ciencia conciencial que se está gestando contemporaneidad. El camino metodológico por el que transcurre la investigación es la Sistematización de Experiencias. De las experiencias sistematizadas emergieron tres claves teóricas fundamentales para la reflexión en el contexto de la educación universitaria, las cuales son: (a) pensamiento científico renovado; (b) educación e investigación con carácter existencial y; (c) de la metodolatría a la metodología científica.

Palabras Claves: investigación; educación; formación; investigadores; ciencia

Abstract: the formation for scientific praxis of the educational researchers of our University is developed under the positivist mentality, promoting an educational culture of the ironical instrumental and recipes logic. It is exposed the serious epistemological crisis, an ontological and anthropological vacuum when it comes to understanding reality. This research approaches existentially to the epistemological keys that can contribute to think the formative processes and the scientific praxis of the educational researchers to inscribe them in the way of the new consciential science that is being born contemporaneously. The methodological path through which the research takes place is the Systematization of Experiences. From the systematized experiences emerged three key theoretical keys for reflection in the context of university education, which are: (a) renewed scientific thinking; (b) education and research with existential character and; (c) from methodolatri to scientific methodology.

Key words: research; education; training; researchers; science; customer service; organizational culture; corporate philosophy; positioning; taxi cooperatives 


\section{Introducción}

Los signos de los tiempos nos están ubicando como testigos y actores excepcionales de una nueva racionalidad científica; es decir, un tiempo nuevo, el tiempo de la vida, que se pregunta ahora por el sentido de la totalidad y plenitud de la existencia. Ya no es tiempo del

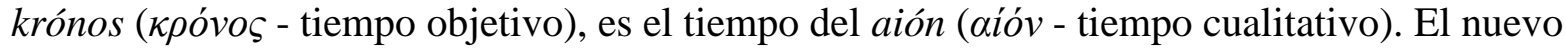
tiempo científico es de la nueva narratividad postmoderna (Lyotard, 1987), marca el discurso de la construcción de los nuevos conocimientos científicos y el quehacer humano.

La postmodernidad se caracteriza por una profunda crítica que busca hacer emerger una epistemología y una ontología adecuada para nueva manera de alcanzar el conocimiento del ser. La racionalidad científica emergente se abre a la comprensión de la existencia del hombre en su relación con el mundo, de las estructuras y conexiones que le dan significado vital, de una ciencia que se encarna para aproximarse más de cerca a la esencia de la verdad, como nuevas tareas de meditación de la dinámica de la generación del conocimiento.

Es nuevo tipo de reflexión que reconoce la condición humana y las consecuencias gnoseológicas para la reforma del pensamiento científico, donde no es posible ningún control metodológico, sino la meditación profunda sobre los fundamentos onto-antropo-epistemológicos del mundo de la vida, donde transcurre la vida de los hombres, el cual se constituye en la fuente ineludible del nuevo quehacer científico. En este sentido, la racionalidad teórica postmoderna es una dura crítica de deslegitimación de la hegemonía de la modernidad. Ésta ha tenido consecuencias epistemológicas y metodológicas serías para la investigación científica en el campo de las ciencias humanas y sociales; sus ideas se desarrollan de manera relevante desde la perspectiva cuantifrenética, donde la vida es un concepto hueco y sin sentido para la ciencia y la investigación tradicional. Ocurriendo así un vacío ontológico y antropológico de la realidad.

La modernidad es una racionalidad que no satisface las ansias de sentido de los hombres. El discurso moderno que se traduce en la praxis científica que vacía al mundo de su naturaleza ontológica y antropológica, que de fondo no es más que la ausencia del elemento epistemológico de comprensión y significación fundamental (García, 2000). Como consecuencia, La pregunta por el sentido y el destino de la vida del hombre y de éste, en el mundo resulta implanteable, es un absurdo. En la educación esta situación tiene su origen en los planteamientos de la pedagógica moderna que se caracteriza por el realismo, que se insubordinó contra el "formalismo humanista" (Gadotti, 1998: 71) pregonando la superioridad del dominio del mundo exterior-empírico sobre el mundo del interior-sujeto. De humanista, la educación se convirtió en científica donde el conocimiento sólo tiene sentido cuando se prepara para la acción (dimensión instrumental).

La des incardinación progresiva de lo humano de la ciencia y de la educación ha llevado a la crisis y la emergencia epistémico-paradigmática. La ciencia y la educación que están bajo el paradigma científico, que niega al sujeto y su existencia en el mundo, deben ser un motivo para pensar los principios de la formación de los nuevos profesionales e investigadores de la educación que se está gestando en el seno de la Universidad. A pesar de los esfuerzos de ruptura epistemológica, en tres de las principales universidades venezolanas, reconocidas por la formación de docente e investigadores educativos, como son la Universidad Pedagógica Experimental Libertador (UPEL), Universidad Simón Rodríguez (USR) y la Universidad 
Nacional Experimental Rómulo Gallegos (UNERG); particularmente en el área de la producción de Postgrado destaca como aspecto común la presencia predominante del perfil epistémico positivista (Bermúdez, 2016). Esta situación se aprecia en la praxis pedagógica y la aplicación metodológica del proceso de formación. A continuación, describimos cada uno de los aspectos señalados:

Praxis pedagógica y curricular: los planteamientos de la racionalidad positivista se constituyen en un instrumento de cultura educativa a través del curriculum, que se tramite a través de los docentes y alumnos-investigadores. Los planteamientos positivistas se han enraizado pedagógica e históricamente a través la formación científica, a tal punto, que dicho paradigma adquiere coordenadas divinas, producto de la no racionalización del mismo. Tal realidad es confirmada por el investigador educativo Zaá (2012): La formación de pregrado y postgrado se caracteriza por el hábito de realizar investigaciones bajo el paradigma positivista, pero en una forma mecánica o recetaria de hacer ciencia, sin el suficiente conocimiento epistemológico, puesto que no se manejan las corrientes filosóficas, enfoques paradigmáticos y posturas que rigen los procesos del pensamiento para construir ciencia.

Los investigadores son sometidos a procesos pedagógicos de formación acríticos. Las estrategias didácticas configurarán una "repitencia" de conocimientos que se inscriben dentro del enfoque cuantitativo, se impide la apertura a otros enfoques, esto resulta un salto cualitativo hacia atrás, hacia el paradogma. Se ha creado una "conciencia acrítica y reducida, tanto en el aula como en los docentes que animan y evalúan las investigaciones de grado que se conforman con el simple conocimiento de pasos y procedimientos para realizar investigaciones partiendo del enfoque en cuestión” (Bermúdez, y García, J. 2008, p. 113-114).

Además, la investigación a la que se tiene acceso en las bibliotecas de nuestras universidades son aquellas que reproducen la lógica instrumental de la racionalidad positivista moderna. La mentalidad moderna hace suyo las fuente y lugares que acercan al conocimiento. Así lo deja ver Sánchez (1998) cuando afirma explícitamente: La mayor parte de los textos de publicación sobre el tema se centraliza generalmente en cuestiones técnicas y metodológicas, muchas veces tratadas bajo ópticas positivistas, que siendo una de las tendencias predominantes en la investigación educativa, considera inútil la discusión sobre diversos abordajes metodológicos y tendencias epistemológicas, pues admiten un único método científico, aquél que sigue el estatuto de la «ciencia positiva», los procesos empíricos-analíticos y las reglas del discurso hipotético-deductivo (p. 13).

Metodologías de investigación: la práctica científica, como requisito académico, se caracteriza por un monismo metodológico propio del método cuantitativo, y ésta reducen a una simple aplicación de técnicas e instrumentos de recolección de datos y análisis estadísticos.

La investigación se reduce a una simplificación racionalista. El proceder científico es mecánico y plagado de normas. Son procesos de investigación desnaturalizados y deshumanizados, angustiantes y sin sentido real para el investigador. Persiste en las investigaciones la linealidad y la dura lógica metodológica. Traspolan la lógica positivista en las investigaciones de carácter cualitativo, lo cual, de fondo deja ver que lo que hay es una linealidad que responde a los planteamientos clásicos de investigación. Denotando una falta profunda de 
reflexión sobre los principios y la formulación práctica del nuevo paradigma. Reina una superficialidad ingenua que riñe con el espíritu de la investigación cualitativa. La razón de ser de las investigaciones de carácter educativo se reduce al uso de estrategias e instrumentos para recolectar y verificar estadísticamente datos, de manera aislada y descontextualizada olvidando la cuestión del sentido, del qué es. La preocupación se centra más en las metodologías, en el cómo, que en la naturaleza de la realidad problema de estudio.

Esta doble descripción, desnuda al discurso moderno en la formación científica de los investigadores educativos que se forman en nuestras universidades. Se ha constituido en una educación del orden y del equilibrio, reduccionista de la realidad y en una ciencia encerrada en sí misma, deshumanizada y deshumanizadora. No penetra ni resuelve los grandes dilemas espirituales del hombre y el cosmos, no puede ir más allá de lo aparente para vivirlo, para comprenderlo y luego comunicarlo de verdad.

Esta situación ha llevado a un de desencantamiento de los procesos educativos en la formación científica de los investigadores educativos. Aquí radica el punto de quiebre, de emergencia y revolución epistémica hacia algo nuevo para la ciencia y la investigación, que todavía persiste bajo el tutelaje del discurso de la modernidad. En este sentido, la experiencia investigativa ha hecho emerger una preocupación que nos ha llevado a preguntarnos ¿sobre cuáles nuevos senderos epistemológicos debe discurrirse para pensar los procesos de la formación y de la praxis de la investigación científica educativa?

La respuesta a este interrogante queremos encontrarlo siguiendo los senderos de la nueva racionalidad que procura una ciencia más humana, vivencial, conciencial y contextual. Una ciencia que se aproxima a la existencia del hombre y a su relación con el mundo, a las estructuras y conexiones que le dan significado vital, que se encarna para aproximarse más de cerca a la esencia de la verdad para comunicarse con su propio lenguaje. En tal sentido, desde la nueva racionalidad emergente se brinda una posibilidad novedosa de acercarnos y reflexionar sobre la situación problemática, tal como es vivida por los investigadores, como les agobia y les fatiga durante todo el proceso de investigación científica. El punto de partida son las vivencias de los investigadores educativos, tal como es comprendida en profundidad en su universo subjetivo (Van Manen, 2003).

Las experiencias vividas por los investigadores educativos sustantivos de nuestras universidades constituyen la fuente primaria original desde donde pensar lo que serán los nuevos significados epistemológicos, dinámicos por su carácter existencial, que servirán para orientar lo que serán los nuevos senderos por donde deberá transitar la formación y la praxis investigación científica educativa. En este sentido, el propósito de la investigación es acercarse existencial y sistemáticamente, a partir de las experiencias vividas de los investigadores educativos sustantivos, a algunas claves epistemológicas que puedan contribuir a pensar los procesos formativos y la praxis científica de los profesionales de la educación que se lleva a cabo en la Universidad, para inscribirlos en el camino de la nueva ciencia emergente. siguientes:

El proceso de investigación se estructura en cuatro momentos fundamentales que son los 
1. La Investigación educativa y la formación científica: se desarrollan los aspectos teóricos de la evolución de la investigación educativa y lo que han sido implicaciones en la formación e investigación científica.

2. El camino metodológico: se presenta el método, los momentos medulares de la Sistematización de las Experiencias y los informantes sustantivos que participaron en la investigación.

3. Lectura de las experiencias sistematizadas: se presentan las tres claves teóricas que emergieron del proceso de teorización y que se revelan como elementos fundamentales para pensar la nueva formación científica de los investigadores educativos.

4. Consideraciones finales: se ofrece en razón de las experiencias una reflexión sobre la necesidad de nuevo discurso científico y de nuevos sujetos comprometidos vitalmente con la investigación educativa.

\section{La Investigación Educativa y la Formación Científica}

La investigación educativa vive hoy una situación paradójica, está en un proceso de transformación al igual que toda la ciencia moderna. Esta realidad encuentra su explicación en los fundamentos que le dieron origen y orientaron su productividad científica. En tal sentido, nace en el siglo XIX con el nombre "Pedagogía Experimental Educativa", una disciplina empírica marcada por los códigos epistemológicos del positivismo reinante. La investigación se realizaba bajo la influencia positivista para dotar de cientificidad a la disciplina naciente.

La investigación educativa nace en el contexto histórico-social de la modernidad donde el pensamiento filosófico positivista resalta el interés por afianzar la educación sobre fundamentos empíricos y cuantitativos e incorporar el método experimental en las ciencias humanas (Delgado, 2011). Emerge permeada por los postulados de la modernidad. Los procesos de la formación e investigación educativa, desde su nacimiento, adoptaron la metodología científica objetivista como instrumento fundamental para constituirse en una ciencia (Flores, 2005).

Posteriormente, la investigación educativa se constituyó durante casi todo el siglo XX en una disciplina empírica que privilegió la objetividad del conocimiento, el determinismo de los fenómenos, la experiencia sensible, la cuantificación aleatoria de las medias, la lógica y la verificación en el campo de la educación. La investigación y la formación ocurren bajo el mito de los protocolos metodológicos del método científico, donde lo que importa es el orden epistemológico en los procesos de investigación.

Esto es debido a que la racionalidad moderna positivista genera un tipo de ciencia, que funciona sobre unos presupuestos matemáticos-mecanicistas-funcionalistas (GalileoNewtonianos) y en la que lo que revela de la realidad es la cantidad-disyunción (rex extensa Descartes) - y no la calidad-conjunción- a partir de un objetivismo determinista científico natural (Hipotético Deductivo-Comte) (Gajate, 2002).

La asunción del espíritu epistemológico objetivista le acarreó posteriormente duras críticas por parte de los ensayistas de la filosofía contemporánea y los movimientos pedagógicos de la escuela nueva de John Dewey (Gadotti, 1998), debido al agotamiento e insuficiencia del modelo cientificista, los cuales generaron un fuerte impacto sobre las prácticas tradicionales 
debido a que no daban respuestas adecuadas a los requerimientos planteados para la situación y la educación del momento. Como consecuencia, en los últimos lustros del siglo XX, gracias a la crítica epistemológica, se da un giro hacia nuevos códigos epistemológicos para la investigación pedagógica, dejando atrás su condición adjetival y unívoca de experimental, para abrir una mirada hacia el análisis cualitativo, de los significados y sentido de la vida social, alejándose de los planteamientos cuantitativos y de la psicología conductista (Delgado, 2011).

De este modo, nace la contemporánea calificación de "investigación educativa” que parte de la condición humana, del sujeto y su existencia en el mundo, en cuanto ser situado que le da sentido humano al mundo social y educativo. El mundo educativo no puede continuar explicando desde una férrea lógica-instrumental que desconoce la complejidad humana, social y educativa como realidades del hombre. La investigación educativa contemporánea evoca el paradigma moderno, su praxis se reduce al uso de estrategias e instrumentos para recolectar y verificar estadísticamente datos, de manera aislada y descontextualizada olvidando la cuestión del sentido, del qué es fundamental: la significatividad (Salcedo, 2011). La preocupación se centra más en las metodologías, en el cómo, descuidando la naturaleza onto-antropológica de la realidad problema de estudio (Delgado, 2011). El qué-es-fundamental no es materia de reflexión ni de significación. Triunfa la eficacia del medio en razón de los objetivos prefijados (Feyerabend, 2002).

Pero, estudios recientes revelan que la investigación educativa sufre gravemente las consecuencias de los códigos epistemológicos y lingüísticos de la modernidad (Bermúdez, J. y García, J., 2016). Los procesos de formación y la praxis científica de postgrado están minados por los códigos del paradigma decimonónico. La investigación educativa adolece de la normalización científica debido a la insuficiencia de los códigos epistemológicos. Está minada del pensamiento y el lenguaje del pasado científico. Es una rémora que la continua atrayendo hacia el pasado científico que le impide deslegitimarlo.

Las experiencias formativas y científicas adolecen de riqueza porque no se está interpretando debidamente la realidad, sino simples repeticiones que legitiman a la vieja mentalidad. La investigación educativa sufre de la necesidad de un nuevo lenguaje, un lenguaje de carácter vivencial. El lenguaje se constituye en un límite para vivenciar. Mientras se continúe traspolando el lenguaje del viejo paradigma en los ensayos de investigación educativa dentro de la modalidad cualitativa el conocimiento se minimiza. Como somos puro lenguaje y al desconocer su estructura íntima y vital, su semiología, todos los esfuerzos científicos por integrar y acercarse a la realidad, a los mundos de vida del hombre concreto, de una manera vivencial y holística, o sea, para experimentar y vivir en plenitud toda su riqueza, se carece de significado. (Bermúdez, J. y García, J., 2016, p. 146)

El reto epistemológico contemporáneo de la formación e investigación científica es lingüístico. La carencia de la racionalidad positivista para la ciencia radica en su insuficiencia lingüística. Miente a través de un lenguaje racionalista que hace creer al hombre que la razón objetiva es la única facultad para entender y dominar al mundo. Es una racionalidad que se comunica con un lenguaje que pierde toda pasión e interés existencial en el pensar del propio sujeto y en el existir de los otros. Por tanto, es una completa devaluación del sujeto frente a su mundo y un proceso crónico de deshumanización (Marquez-Fernández, 2011). 


\section{El Camino Metodológico}

El enfoque metodológico que se utiliza en este estudio es el de la investigación cualitativa porque se vincula intrínsecamente con las experiencias vividas de los actores involucrados. Permite sistematizar el conocimiento de forma más humana porque se privilegia la comprensión en profundidad de las experiencias vitales de los informantes sustantivos a través de su universo subjetivo, acerca de los significados de las vivencias que nacen de su modo de vivir e interrelacionarse con el mundo.

En tal sentido, desde lo metodológico, se considera como camino de teorización de la investigación a la Sistematización de Experiencias tal como la propone Barrera (2010), como expresión del ejercicio del pensamiento reflexivo y del proceder instrumental delos investigadores para la generación de la teoría. La elección del camino de teorización se debe a que se vincula con la naturaleza y los aspectos onto-gnoseológicos, profundamente subjetivos, que orientan la intencionalidad investigativa, ya que su cercanía con las bondades epistemológicas de la fenomenología y la hermenéutica que favorece el acceso al conocimiento de las experiencias de los informantes sustantivos e investigadores educativos involucrados, para luego describirlas y pasar a su sistematización.

La Sistematización de Experiencias se constituye en un camino donde encontramos informantes sustantivos y pensadores-investigadores en ejercicio pleno de subjetividad, fuentes originales de conocimiento-propiciador de la descripción del fenómeno, de la experiencia vital; tal como aparece y se hace presente a la percepción. En esos tramos del camino, donde estuvo presente, la subjetividad de las personas (informantes e investigadores) que posibilitaron las claves de comprensión de la esencialidad fenomenológica de las experiencias descritas. Para llevar a cabo el proceso del análisis de las experiencias de vida de los informantes sustantivos se transitó por los tres momentos medulares y fundamentales de la Sistematización de Experiencias que llevaron al proceso de teorización: (a) descripción de la experiencias (registro y procesamiento); (b) abstracción teórica (identificación de las ideas-fuerzas y estructuración argumentativa del corpus teórico); (c) análisis (critica de la teoría emergente).

Los informantes sustantivos se les denomina así por su reconocido papel académico en el área de pregrado y postgrado en educación, los cuales fueron seleccionados de tres importantísimas Universidades del Estado venezolano, destacadas en el campo de la formación docente y de investigadores, las cuales son: (a) La Universidad Pedagógica Experimental Libertador; (b) La Universidad Nacional Experimental Rómulo Gallegos; y (c) La Universidad Experimental Simón Rodríguez.

\section{Lectura de las Experiencias Sistematizadas}

Del proceso de abstracción teórica de las experiencias, que contiene el conjunto de ideasfuerzas y principios que permiten apreciar el sentido explicativo de la construcción de la trama teórica-argumentativa de las experiencias descritas, reveló que el problema de la nueva investigación científica radica en la necesidad de una ciencia de la comunicación fenomenológica de la existencia en el mundo. 
Las experiencias sistematizadas de los investigadores educativos nos introducen de manera nueva en la trama del conocimiento, desde nuevas perspectivas que proyectan el camino hacia una formación e investigación con carácter conciencial, es decir, que toma en cuenta las dimensiones fundamentales de la condición humana, subjetividad y espiritualidad, que dentro de la investigación objetiva no tendrían razón de validación científica. De las experiencias sistematizadas emergieron tres claves teóricas fundamentales, las cuales están en estrecha relación trasversal y determinan el proceso de la educación científica, las cuales son: (1) pensamiento científico renovado; (2) educación e investigación con carácter existencial; (3) de la metodolatría a la metodología científica.

\section{(1) Pensamiento científico renovado}

La experiencia sistematizada sugiere como primera tarea desvestir al pensamiento científico de la vieja mentalidad. Es necesario un ejercicio filosófico que provea a los investigadores educativos de nuevas categorías de pensamiento desde donde cuestionar y pensar la trama de la generación del conocimiento, que proyecten el camino hacia una investigación científica encarnada. Un tipo de pensamiento que desintegra y reducen su objeto de pensamiento a la pura materialidad no comprenden la compleja realidad ontológica del ser relacional en el mundo de la vida. Hace imposible que las experiencias científicas se traduzcan en una comunicación sustantiva de la esencia humana que se conviertan en experiencias vitales para quienes las conocen.

Las experiencias sistematizadas señalan con carácter imperativo un nuevo tipo de pensamiento para la ciencia, con categorías renovadas. Consideran que se deben tener en cuenta las siguientes:

(a) La ciencia es unidad:

La ciencia es una unidad o no será ciencia. Esto conlleva dar un sentido humano a la ciencia. Lo humano se comprende como un conjunto complejo de dimensiones constitutivamente unidas. El reconocimiento del hombre total, como un ser complejo (lo que está tejido junto), es el camino para el que-hacer científico que comporta replantear el espíritu una ciencia unida y no dividida. Reconvertir la ciencia y la investigación pasa por reconocer la unidad de la ciencia, pero esto será posible reconoce primero ser humano total. No se puede seguir con una ciencia que sólo investiga la dimensión objetiva del hombre, olvidando su esencia subjetiva e intersubjetiva, éste no es sólo materia. La investigación científica debe declarar la unidad del hombre, éste es el primer peldaño, el acto principal de la nueva educación científica.

La superación del divorcio histórico entre las ciencia naturales y las humanas que tiene subsumida a la humanidad en una crisis global y que pone en riesgo al hombre y su mundo, no es cuestión de un plumazo, es una transición generacional que ira ocurriendo en la medida que se conecte con un nuevo tipo de pensamiento que cree en la integración entre las visiones de la ciencia y cree que no hay separaciones gnoseológicas a la hora de conocer la estructura de la realidad, piensa que no hay separación ni diferencias, ve la unidad de la realidad.

Superación de la dualidad sujeto-objeto:

Las consecuencias de la práctica del viejo pensamiento han acarreado un tipo de conocimiento fragmentador. Para poder pensar la praxis científica dentro de nuevas coordenadas pasa necesariamente por la superación histórica del dualismo cartesiano. Consideran que es una 
tarea filosófica y pedagógica a la vez. Filosófica, porque el pensador educativo penetrando y adentrándose en la esencia de la realidad la contempla como una unidad, por la vía subjetiva o por la vía objetiva. También, pensándola de otra manera, dentro nuevas categorías epistemológicas y de lenguaje que den pasó a la comprensión de la unidad. La primera tarea del pensamiento es comenzar a pensar que no hay diferencia entre sujeto y objeto, ni siquiera para hacer ciencia. Entre ellos hay una relación significativa.

Pedagógica, en los ambientes académicos se debe abandonar la concepción teórica dicotomizadora que permea la ciencia estableciendo que el proceso de producción del conocimiento es fruto de una relación dual, que separa, en categorías gnoseológicas de sujeto y objeto. Sólo es necesario que sea conocida como historiografía de los sistemas epistemológicos del pensamiento filosófico.

No puede haber para la ciencia fronteras entre el sujeto y el objeto, éstos se confunden, es imposible desvincularlos, ya no se comprenden como dos partes, sino como un todo integrado. El mundo para el hombre es una unidad y él es sujeto de conocimiento en la unidad, no acepta separaciones en categorías racionalistas. La tarea educativa y científica se debe orientar a desvestir al pensamiento científico de la nefasta visión dualista cartesiana para ir construyendo progresivamente un sistema filosófico provisto de categorías epistemológicas de lenguaje que hable en clave de unidad e interrelación entre hombre y mundo, humanidad y mundanidad, subjetividad y objetividad. El conocimiento debe ser un proceso que evoca unidad e interrelación, no lo contrario, donde la realidad y el investigador se comprenden en una relación compleja, dinámica e intercomunicada.

(b) Tetragrampatron epistemológico

Dentro de una racionalidad que reconoce la condición humana emergen categorías las de pensamiento como son: la subjetividad, la intersubjetividad, la cotidianidad y los saberes como elementos fundamentales que conforman el nuevo patrón epistemológico, todos necesarios pensar y desarrollar los procesos de investigación.

La subjetividad, como dimensión esencialmente humana, es una forma de conocimiento. Es camino de teorización y método de observación científica. Es una fuente de conocimiento que se origina en las vivencias, es profunda y no está atada ningún método y es la vía para superar la dualidad deshumanizadora de la escisión sujeto-objeto que pervive en la ciencia. Por esta razón es la posibilidad de una ciencia más humana, donde es posible que el hombre se comprenda como sujeto que forma parte del mundo y se reconozca también en el otro, que es para éste una unidad orgánica del conocimiento. El investigador no puede deslastrarse de la subjetividad. Le introduce en mundos inexplorados hasta ahora para la ciencia. Permitiendo apreciar profundamente el espíritu humano y el espíritu del mundo hasta ahora irreconocible por la ciencia objetiva. Lo adentra en una ciencia de carácter conciencial que hace de lo humano, de lo cotidiano, lo espiritual, lo inmaterial, lo cultural, lo social, lo vivencial y lo experiencial sus contextos más preciados de pensamiento.

La intersubjetividad, dese esta se comprende como un ser relacional, de la alteridad, de la complementariedad. Se constituye en una herramienta para profundizar en la realidad, en una actitud científica, en vista que la pluralidad de visiones de los investigadores encuentra en la 
complementariedad un camino abierto para que puedan intervenir y hacer aportes significativos a los procesos de construcción del conocimiento, dando pie a una ciencia más unidad y comunicada a través de esfuerzos interdisciplinarios, multidisciplinarios y trasdisciplinarios. La intersubjetividad, lega posibilidad de la alteridad para un diálogo fructífero y enriquecedor entre los investigadores, sea cual sea su visión de ciencia y su particular área disciplinar del saber, pues, los abren a nuevas y diversas formas de ver sus procesos particulares de investigación.

La cotidianidad es fuente de cientificidad, hasta ahora no tenía ningún significado para la racionalidad positivista. En ella se configura y expresa el ser del hombre concreto, donde lo esencialmente humano es científico. La cotidianidad es parte del credo de la vida del investigador que aprecia desde la subjetividad una investigación científica vivencial. Reconocer la cotidianidad conlleva a una mayor apreciación del ser del hombre concreto desenvolviéndose en su mundo de vida. La cotidianidad es el mundo de vida, permite ver al hombre de piel, de carne y hueso, que se alegra y sufre, que trabaja y disfruta, que vive y sueña, que nace y muere; acercándonos de diferente manera más al ser de los seres humanos. La cotidianidad es el ser de una investigación científica vivencial. La cotidianidad es el ser de las personas, permite ir más allá.

Los saberes son la expresión gnoseológica del mundo de vida del hombre de a pie, de carne y hueso, del ser que emerge y que no puede ser reducir a categorías dualistas o exclusivamente racionalistas. El conocimiento da paso a los saberes que son en un sentido total la expresión significativa particular-local de todas las dimensiones constitutivas que marcan la forma de hacer y pensar de los seres humanos que se configuran en sus mundos de vidas y de la interrelación con otros mundos de vida. Los saberes son la expresión epistemológica del ser y del hacer del hombre concreto que es producto de la complejidad de su mundo de vida y de la interrelación con otros.

(c) Nueva narratividad científica:

Caminar por los senderos de un nuevo pensamiento científico significa hacerse de nuevas categorías. La nueva ciencia necesita de un nuevo lenguaje. La investigación y la formación científica carga sobre sus hombros el lenguaje de la ciencia moderna. Es un tipo de lenguaje que se constituye en un límite para aprehender la realidad en su estado natural. La tarea de los pensadores educativos es adentrarse en la búsqueda de un nuevo lenguaje. De un lenguaje para vivenciar y atrapar el significado de las experiencias. Un lenguaje que nos haga capaces de penetrar en la semiología del mundo de vida del hombre concreto, para experimentar y vivir con él, siendo parte con él de un proceso vivencial cargado de significatividad. Es necesario un nuevo lenguaje que nos introduzca en una nueva gnoseología, en una nueva relación gnoseológica suficiente para vivenciar, en un lenguaje para atrapar el ser, la esencialidad de los significados para los hombres en su interrelación con sus mundos de vida.

El lenguaje es para la ciencia la posibilidad de una nueva narratividad científica, no habrá posibilidad de una ciencia nueva si no se introduce en la dinámica de las leyes del lenguaje. La investigación debe buscar en la cotidianidad y la subjetividad del hombre concreto las reglas configuradoras del código lingüístico, que determina las distintas jugadas de los juegos del lenguaje, por el cual éste genera sus saberes y el significado para su vida. El problema del lenguaje es determinante para el investigador educativo. Éste debe ser un hermeneuta. Debe 
hacer uso de la hermenéutica como una práctica fascinante porque le introduce en un proceso de la comunicación fenomenológica-encarnada, de la significatividad más profunda del espíritu del hombre.

\section{(2) Educación e investigación con carácter existencial}

Las experiencias sistematizadas creen que la investigación debe transitar por nuevos senderos. Este caminar no es una tarea fácil, no estamos formados para ella. La racionalidad emergente necesita de nuevos pensadores científicos. Pero, las experiencias muestran la insuficiencia de nuestra educación científica para generar un tipo de pensamiento y un lenguaje para vivenciar y contextualizar la comprensión de la realidad. La nueva mentalidad está en estado de crisálida, todavía una tarea, no se puede a la suerte del azar.

Como segundo compromiso, la tarea es educativa, aunque se han ido adquiriendo aisladamente algunas herramientas epistemológicas importantes en la medida que se ha dado un acercamiento a teorías que propugnan un cambio de racionalidad. Reconvertir la investigación científica pasa por repensar su relación con la educación. El primer paso hacia la nueva ciencia pasa por devolver a la educación su sentido original. No es una práctica de poder, es un proyecto civilizatorio. Las experiencias señalan algunas claves configuradoras de la nueva educación para la formación científica.

(a) Educación e investigación para la humanización:

La experiencia de los investigadores considera la necesidad de repensar la educación como un proyecto civilizatorio. La educación es sobre todo la base y el eje fundamental de humanización, de transformación del hombre y su mundo. La educación ya no puede concebirse como una práctica interesada, una esclava de la sociedad y la ciencia.

La educación es el contexto vital previo de edificación y configuración del espíritu humano que busca comprenderse a sí mismo en su relación con el mundo para la transformación y el cambio. La experiencia de los investigadores subraya la necesidad de pensar la educación como el contexto global que permea sobre cada ser humano para nutrirlo en el ejercicio de la complejidad de la vida. Pensar la educación como marco general del proyecto civilizatorio es concebirla como la base fundamental que configura y comprende al hombre total, lo incorporando con todas sus condiciones, no sólo en el plano cognitivo. La educación adquiere un significado distinto. Se educa para vivenciar y comprender el significado del ser de la realidad. La educación es para vivenciar y comprender el significado de lo vivido, es una experiencia vital de carácter conciencial.

Educación e investigación se encuentran. Se establece una relación de necesidad biyectiva. Se educa para vivenciar y comprender y se vivencia y comprende para investigar. Educación e investigación, son un sólo camino búsqueda del significado del ser humano, que nacen de las experiencias más genuinas y cotidianas de la vida, la primera es la vía para el encuentro y el significado como seres en el mundo y la segunda ve en él las huellas para el encuentro y el descubrimiento del significado del ser. Educar e investigar son experiencia reflexiva y transformadora. 
La educación hace a la investigación y la investigación hace a la educación. La educación y la investigación ya no pueden ser lo mismo. Educar e investigar deben ser una forma de vida en la búsqueda del significado y la transformación. No pueden ser una práctica cognitiva e instrumentalizada y mucho menos un requisito académico que se ejecuta como un imperativo cargado de desánimo y desaliento para obtener un ascenso, un grado o un mejor sueldo. La educación entendida como puro pragmatismo académico es una forma de mentir.

(b) Educación e investigación basadas en una racionalidad humana:

La experiencia de los investigadores muestra que tal práctica formativa deja ver que ni la educación, ni la investigación, en el momento coyuntural del pensamiento científico, son comprendidas, y mal que bien, mal llevadas en el campo educativo de la formación científica e investigativa.

Esto conlleva cuestionar la tradicional práctica educativa, el discurso científico moderno que la fundamenta. Hacer un cambio progresivo de la educación pasa por el cuestionamientodel viejo paradigma, insuficiente por sus caracteres simplificacionistas, reduccionistas, fragmentado, controlador, hiperespecializado, objetivista e incomunicables con la esencia espiritual del ser humano. Impide comprender al ser humano y su mundo como una unidad compleja. Una educación anclada en el viejo modelo no forma para la humanización. Se agotó, sus fronteras ya fueron traspasadas. Ya no se puede educar al ser humano como si fuese un objeto. Debe ser comprendido y educado desde otros puntos de vistas, que reconozca la complejidad del ser humano. Se plantea entonces la necesidad de nuevas categorías de comprensión, de una nueva gnoseo-epistemología, un nuevo pensamiento y un nuevo lenguaje para la educación y la investigación, que se adecue con la esencia humana. Donde, el ser humano deja de ser objeto para ser sujeto, persona y espíritu.

En este sentido, las experiencias consideran que las circunstancias contemporáneas del pensamiento científico ofrecen una serie de experiencias novedosas de reflexión, como son la fenomenología, el pensamiento complejo, la hermenéutica y la teoría socio-crítica que brindan nuevas herramientas epistemológicas que ofrecen un tipo de pensamiento más cercano a la naturaleza de la realidad. Sus peculiaridades apuestan por el ejercicio de una racionalidad vivencial, de carácter existencial y complejo. Estas experiencias del pensamiento permiten reconocer todos los elementos imbricados en la vida, en la realidad, la complejidad y las relaciones de los sujetos que hacen el mundo educativo y sus mundos de vidas, no solamente en un plano cognitivo y político, en el que estamos absortos dentro de la vieja racionalidad.

Un nuevo pensamiento para la educación la replantea y la redimensiona, no solamente la educación y la investigación de los educadores, ni la formación docente, lo cual sería un reduccionismo, sino la de todas las áreas del saber. Donde la educación y la investigación son una tarea humanizadora que integran todas las áreas del saber para superar la fragmentación deshumanizadora. Educación e investigación trasversalizando y permeando el proceso formativo para vivenciar-comprender. Esto conlleva a una recomprensión de los procesos educativos en general y de la investigación científica dentro ellos, para que reconozcan al ser humano y lo comprendan en su complejidad de vida, en su todo vital. Se solicitan una educación e investigación para vivenciar y comprender, totalizadora, que impregne todas las áreas del saber, 
y no sólo, la de la formación de los educadores. Una educación e investigación como forma vida, una racionalidad de la pasión y la razón.

(c) La investigación educativa eje de formación profesional:

Las experiencias señalan que se está fallando horriblemente. Hasta ahora la investigación educativa se reduce a la formación docente, a una asignatura del pensum de estudios, y a desarrollar "una investigación", muchas veces no sabe de qué, conforme a una metodología. La investigación educativa como una asignatura del pensum de estudio no existe. Tampoco es una propiedad exclusiva de la formación docente, ni de los docentes universitarios. Continuar con esta práctica sería un reduccionismo absurdo que seguirá ensanchado la incomprensión del significado de la investigación educativa en la formación general hasta la profesional. Los pensadores educativos, desde la actitud de cuestionamiento y revisión, consideran que la investigación educativa debe ser pensada como el eje fundamental de la formación general y profesional, que se inserta en todas las ramas del saber científico, transformando los procesos de formación, para ser educado conciencialmente, en el área donde se es educado.

Es el eje integrador y transformados de todos los procesos educativos, en todas las áreas del saber, en aras de su mejoramiento y profundización, sin excepción. La investigación educativa en cuanto eje constituye un sistema de relaciones que permite comprender, articular y transformar el resto de los ejes educativos, donde estos son integrados por éste para permear hacia procesos formativos humanizantes es decir, dirigidos dinámicamente hacia una formación trasversalizada, que permitan al educador y al educando ejercitarse dentro de una praxis vivencial-conciencial que le permiten comprender y atrapar el significado de sí mismos en el mundo.

Al considerar la investigación educativa como el eje fundamental, es posible comprender e integrar, en todo proceso educativo general y profesional, la generación del conocimiento desde los diversos ejes de formación y la triada de dimensiones vivencial-conciencialcomprensión, hasta ahora separados. Porque al pensar la investigación educativa, dentro de la educación toda, como el faro desde donde se miran y aplican el resto de los ejes es posible integrarlos como un sistema dinámico educativo de aprendizaje-conocimiento-humanización. Las experiencias creen en la posibilidad de pensar la investigación educativa como el eje fundamental de la formación general y científica desde el cual se educa para la vida y la investigación adquiere un nuevo significado. Es un credo vital, un compromiso sustantivo que se constituye en una forma de vida. Se le asigna un verdadero sentido a la investigación.

La investigación educativa deja de ser una asignatura, un método o una propiedad de los docentes. La cuestión es que todo siempre somos educadores y educandos a lo largo de la vida, por tanto, todos somos investigadores educativos. La investigación se integrar a nuestra vida como un credo fundamental y un que-hacer cotidiano para la transformación de los propios procesos educativos y para la sensibilización en el ejercicio de la praxis profesional con todas sus consecuencias.

(d) Educación e investigación para creer 
La investigación educativa es fundamental, no se concibe un docente que no investigue. Ser docente significa vivenciar y comprender la praxis, pensarla para transformarla y transformar. Un educador es un filósofo, un pensador consciente y con conciencia.

Las experiencias ven con tristeza que la realidad es otra, no se está dando una asimilación en los educandos de la condición docente. No se está formando para una forma de vida, sino para obtener un grado académico en la vida. La situación es dolorosa, la investigación es lo que no es. No existe una reflexión profunda, ni una sistematización de las experiencias. Los procesos fundamentales de la formación no formar para la empatía. La formación tradicional pone límites. El problema no es ya la tesis "Todo Menos Investigación" (TMI), sino que se develado algo peor, es el "Todo Menos Investigadores" (TMI's), no creen en la investigación, no forma parte de su credo vital, no es sustantiva.

Las causas de esto se encuentran en la desintegración de la investigación educativa de todo el proceso formativo, el cual se replica en el ejercicio formal de la docencia. Por el contrario, el significado de la investigación educativa en la formación docente es total, ella es un eje académico y de vida. Sólo así el docente se comprende como un investigador, un pensador, un sistematizador y un socializador de sus propias vivencias, para transformar y mejorar su accionar diario e impactar en las comunidades. El docente es un investigador de su cotidianidad. Por eso, para éste la investigación educativa significa plenitud, significa su propia vida. De aquí, que resulte más fácil formar para lo que no es la investigación educativa que para lo que es.

Entonces, se vuelve más necesario transitar hacia procesos formativos vivenciales que pongan en cuestión todos aquellos procesos académicos que niegan la posibilidad de vivenciar la vida.

\section{(3) De la metodolatría a la metodología científica}

Como tercer compromiso las experiencias sugieren la necesidad de una investigación más cercana a la realidad y las vivencias de los investigadores y no una investigación cercana a la receta. La metodolatría no puede seguir siendo el camino para una investigación significativa.

La investigación recetaría y sus protocolos niegan las vivencias y la racionalidad del investigador al ir en contra de sus propias experiencias, pensamientos y originales esfuerzos de búsqueda del conocimiento de la realidad que él está observando. La metodología-receta impone los límites y fronteras de comprensión. Volver a las bases de la investigación exige desmontar el excesivo recetismo. Las consecuencias de un tipo de pensamiento instrumentalista son nefastas para la investigación educativa. Se vuelve necesario establecer equilibrios, aunque las comunidades científicas no quieran reconocerlo. En este sentido, se ofrece algunas categorías de reflexión para la comprensión de la metodología.

(a) Los guardianes del saber:

La consideración de la investigación científica como poder y control es uno de los principales obstáculos para la reconvertir la investigación científica. El poder es ejercido por las comunidades científicas que han nacido del legado del objetivismo científico. Los pensadores educativos califican peyorativamente a sus miembros como "los guardianes del saber"; en razón de que parecieran ser los poseedores de toda la verdad y los encargados de determinar la ciencia oficial. 
Son los principales encargados de mantener las estructuras rígidas e interesadas de las instituciones universitarias, científicas y gubernamentales para la producción y promoción científica. Son los responsables de que se cumplan los requerimientos académicos, metodológicos y administrativos para la consideración del carácter científico de las investigaciones que se llevan a cabo. Es grave el papel que ejercen los guardianes del saber, por su carácter absolutista sobre los investigadores mismos. Se constituyen en una negación de las vivencias, las experiencias, la racionalidad, la espontaneidad y la creatividad de los investigadores. Los investigadores trabajan no para investigar, sino para cumplir con los requerimientos y deseos que establecen sus tutores y evaluadores.

Los guardianes del saber son un freno para los investigadores. Son irreflexivos y dogmáticos. Éstos esperan que los investigadores repitan lo que ellos tienen en mente y enseñan en sus cátedras. Hacen que los investigadores renuncien a propias vivencias, experiencias, esquemas de proceder, racionalizar y creatividad han desarrollado y encontrado. Los investigadores trabajan es para la mentalidad y el esquema que tienen en la cabeza "los guardianes del saber" y nunca trabajan en función de la investigación científica. Los guardianes del saber a través de los procesos de enseñanza, son normalizadores institucionales de la vieja mentalidad objetivista y cuantitativa. En nombre de un supuesto rigor científico imponen la metodología-receta como un dogma. Han vaciado la ciencia y la investigación de su dimensión esencialmente filosófica y humana.

(b) El método es una creación científica

Para superar el excesivo recetismo que ha desprestigiando la razón científica del método, es necesario comenzar a pensarlo como un camino creativo y axiomático. Las puestas en escena de las nuevas corrientes de pensamiento de carácter cualitativo dan más importancia a la originalidad y creatividad de los investigadores. Permiten vivenciar e integrarse con la realidad de investigación, preliminar y originalmente, para luego construir el método. Dan cabida a la observación incontrolada como primer método de investigación, para luego construir el método que calce con la realidad objeto de pensamiento.

El método se debe comprende como un ejercicio de creación científica, nunca de copia ni de imposición, que se ajusta a la naturaleza de la realidad objeto de las vivencias y pensamientos del investigador. Los procesos metodológicos son siempre previamente una praxis donde prima el ejercicio filosófico-vivencial de apropiación del carácter epistemológico y ontológico de las realidades de reflexión. El método es una encarnación necesaria entre el investigador y la realidad de reflexión para alcanzar el conocimiento significativo. El método es importante en cuanto se vincula con las características esenciales de la realidad. Tiene que calzar y vincularse con la realidad que preliminarmente está vivenciando los actores científicos.

La metodología es una dimensión adjetivada a la triada de dimensiones onto-antroepistemológica, es decir, a las vivencias, las experiencias y el discernimiento que hagan los investigadores científicos de la realidad motivo de su pensamiento. La metodología contribuye a ampliar lo que fueron los procesos preliminares de las vivencias de la realidad de estudio. Sirve para penetrar en ella de verdad, para vivirla, para sentirla, para gozarla, para disfrutarla. El método es un ejercicio de consustanciación real. En este sentido, la creatividad científica viene a conformarse una virtud necesaria de la nueva lógica científica. Ella establece el equilibrio entre 
la rigurosidad y la flexibilidad metodológica, agregando a la investigación un nuevo significado a su sentido científico, evitar así cualquier riesgo de metodolatría o de libertinaje metodológico.

\section{Consideraciones Finales}

Las experiencias sistematizadas ven en la nueva metáfora del conocimiento novedosos principios filosóficos para racionalizar los procesos pedagógicos de la formación científica. Se plantean a la educación universitaria como los nuevos fundamentos para la formación de epistemólogos que animen el viraje filosófico hacia el nuevo pensamiento en el proceso de retorno a las fuentes originales de la investigación científica educativa, vivencial y encarnada. Para ello, la formación universitaria debe potenciar espíritus libres y creadores, con valor humano y filosófico que crean en lo que profesan.

En este sentido, son necesarios gnoseó-epistemólogos para un nuevo discurso educativo que puedan adentrarse en una epistemología compleja, la del hombre de a pie, en su vida cotidiana, la cual fluye en la multidimensionalidad de sus experiencias del mundo. Es la hora de la formación científica universitaria postmoderna que desafía el abordaje de la humanidad en el mundo con toda su complejidad, al hombre en su cosmos, con sus cualidades y complejidades, sin reducirla ni estereotiparla, superando la férrea lógica instrumental.

Podemos finalizar afirmando la necesidad una educación que forme filósofosinvestigadores de la ciencia de la educación, autoconciencias críticas comprometidas vitalmente. El proceso educativo debe hacer emerger sabios y científicos comprometidos existencialmente, espíritus con una espiritualidad creyente, sujetos capaces de autoconocimiento del logos que está en movimiento, del hombre en relación con el mundo, como la esencia misma de la existencia humana. El filósofo-investigador de la ciencia hace de su quehacer epistémico un modo de vida, su virtud.

\section{Bibliografía}

Barrera, M. (2010). Sistematización de experiencias y generación de teorías. Caracas: SYPAL.

Bermúdez, J. y García, J. (2008). Congruencia epistémica de los trabajos de grado, en torno a los paradigmas que articulan la investigación socio-educativa de la Maestría en Educación, Mención Educación Superior, de la UPEL Maracay. Entretemas, 10, 89-119.

Bermúdez, J. (2016). Claves teóricas para pensar la formación y la práctica de la investigación de los profesionales de la educación. Trabajo de Ascenso no publicado para optar a la Categoría Académica de Asociado, Universidad Pedagógica Experimental Libertador del Instituto Pedagógico Rural “El Mácaro”, Venezuela

Bermúdez, J. y García, J. (2016). El lenguaje como problema en la investigación socio-educativa. Sophia, 20, 139-159.

Delgado, F. (2011). Paradigmas y retos de la investigación educativa. Una aproximación crítica (2a. ed.). Mérida-Venezuela: Consejo de Publicaciones de la Universidad de los Andes. 
Feyerabend, P. (2002). Contra el Método. Barcelona-España: Folio.

Flores, C. (2005). Investigación educativa. [Documento en Línea] Obtenido de: http://www.monografias.com/trabajos28/investigacion-educativa/investigacioneducativa.shtml. [Consulta. 2016 Enero 21].

Gadotti, M. (1998). Historia de las ideas pedagógicas. Argentina: Editores Siglo XXI.

Gajate, J. (2002). Positivismo, utilitarismo y evolucionismos. Bogotá: El Buho.

García, J. (2000). La dignidad de la naturaleza. Granada: Comares.

Lyotard, J. (1987). La condición postmoderna. Informe sobre el saber. Madrid: Cátedra.

Marquez-Fernández, A. (2011). De la razón sensible a la pasión afectiva. En Valera-Villegas (2011). Formación de la sensibilidad filosófica, arte, pedagogía. Caracas: Decanato de Postgrado de la Universidad Nacional Experimental Simón Rodríguez.

Salcedo, C. (2011). La imposibilidad de ser otro. Inquietud y autenticidad en Agustín a partir del joven Heidegger. Puerto Rico: Publicaciones Puertorriqueñas, Inc.

Sánchez, S. (1998). Fundamentos para la investigación educativa. Presupuestos epistemológicos que orientan al investigador. Bogotá: Magisterio.

Van Manen, M. (2003). Investigación educativa y experiencia vivida. Barcelona-España: Idea Books.

Zaá, J. (2012). Del discurso científico de la modernidad a una nueva narratividad en la construcción del conocimiento. Nexos, 1 (2). Obtenido de: http://nexos.unerg.edu.ve/portal/index.php?option=com_content\&view=article\&id=25:de 1-discurso-cientifico-de-la-modernidad-a-una-nueva-narratividad-en-la-construccion-delconocimiento\&catid=13\&Itemid $=130$. 